\title{
Virtual and Augmented Reality Use Cases for Fusion Design Engineering S.Gazzotti ${ }^{1 *}$, F.Ferlay ${ }^{1}$, L.Meunier ${ }^{1}$, P.Viudes ${ }^{1}$, K.Huc ${ }^{1}$, A.Derkazarian ${ }^{1}$, J-P.Friconneau ${ }^{1}$, B.Peluso ${ }^{1}$, J-P.Martins ${ }^{2}$ \\ ${ }^{1}$ CEA, IRFM, Cadarache, 13108 Saint-Paul-lez-Durance, France \\ 2 ITER Organization Route de Vinon-sur-Verdon, CS 90 046, 13067 St. Paul Lez Durance Cedex, France
}

*Corresponding author: stephane.gazzotti@,cea.fr

As augmented and virtual reality technology is revolutionising the way products are designed and tested, many companies are expanding the range of use cases across their organisations. After 10 years using Virtual Reality (VR) \& Augmented Reality (AR) technology during the life cycle of fusion components, the CEA IRFM has improved its skills developing immersive visualisation scenes for the WEST tokamak [6][5] project and several ITER Site Support Agreement such as Magnet Infrastructure Facilities for ITER (MIFI) [7] and Test Blanket Module System (TBM) [8]. Data preparation process has been optimized, reducing time cost to convert CAD data to an interactive and immersive model. For the WEST Project, VR engineers developed immersive scenes to simulate assembly sequences, to check component introduction using physics and to show $2 \mathrm{D}$ temperature data from diagnostics in a $3 \mathrm{D}$ environment. For the TBM case, health and safety oriented model environment is developed. Features for team simulated human access and hands on operations for As Low As Reasonably Achievable (ALARA) optimisation process with motion-tracking system and virtual avatars with ventilated protective pressurised suit simulating worker protection in nuclear environment. For MIFI application, a specific assembly tool has been developed for TF coils interface located on the top and at the bottom junction. During a first phase, the development was driven by iterative design loop (CAD modelling analysis and cinematic by using VR simulations). By means of interactive simulations and immersive conditions, the interface connection task was validated against feasibility including accessibility. AR has also been introduced in the process since a physical mock-up has been augmented to consider the constraint environment of TF Coils. For TBM application, on site AR simulation of the port Cell equipment will provide enhanced environment description. Collaborative immersive context, digital twins and combination between AR/VR \& physical mock-ups for validation of maintenance since component design phase will constitute challenge for the near future.

Keywords: Virtual Reality, Augmented Reality, WEST tokamak, ITER, Test Blanket System, Health \& Safety, MIFI

\section{Introduction: 10 years of evolution}

Complex environment for Fusion projects such as WEST or ITER tokamak pushes the boundaries of physics and makes technical teams find new ways to think.

In the last decade, CEA IRFM has integrated the use of Virtual Reality in the design process following market technology evolution.

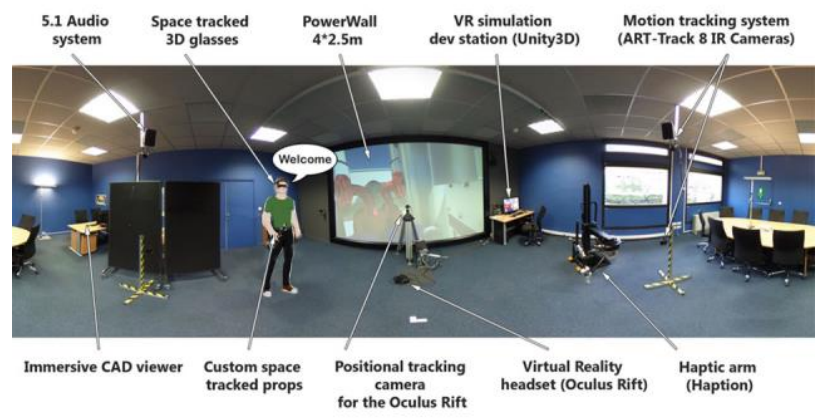

Fig. 1. CEA IRFM VR Room - configuration

In 2017, CEA was able to use VR technologies for immersive studies however, limits were reached such as efficient conversion process from CAD model or limited physical interactions when trying to study specific assembly operations. [1]

Today, boundaries are pushed further using the most advanced XR (eXtended Reality) technologies:
- 6 DoF Virtual Reality headsets such as Oculus Rift $\mathrm{S}$ and Vive Pro Eye

- $\quad$ Augmented Reality (AR) with Microsoft hololens 2 (2019)

- Virtual manikin with motion capture using ART suit (24 points) and LS Suit with Vive tracker technology (8 points)

- Haption haptic arms with force feedback

- High Speed Computer with RTX graphic cards

- Real time hand tracking (Ultraleap Motion)

- Unity software with Interact Plugin [11] using CEA XDE interactive physics engine developed by CEA-LIST

- Pixyz software optimising data process from CAD to FBX 3D models [10]

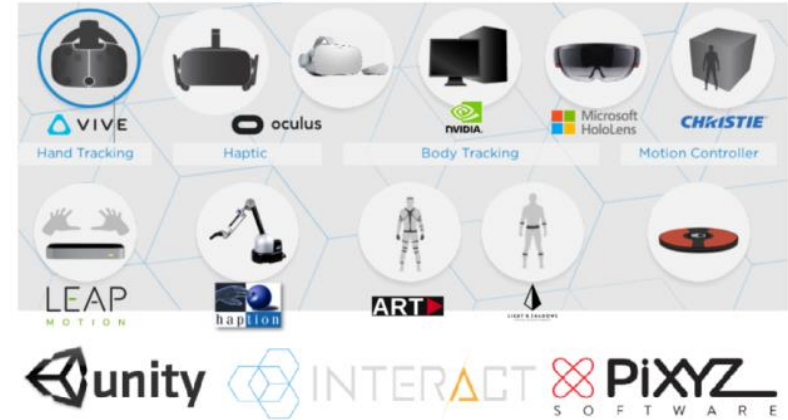

Fig.2. CEA IRFM VR Room - Hardwares \& Softwares 


\section{WEST tokamak}

The Tore Supra tokamak has been transformed in an Xpoint divertor fusion device within the frame of the WEST (W-for tungsten-Environment in Steady-state Tokamak) project, launched in support to the ITER tungsten divertor strategy.

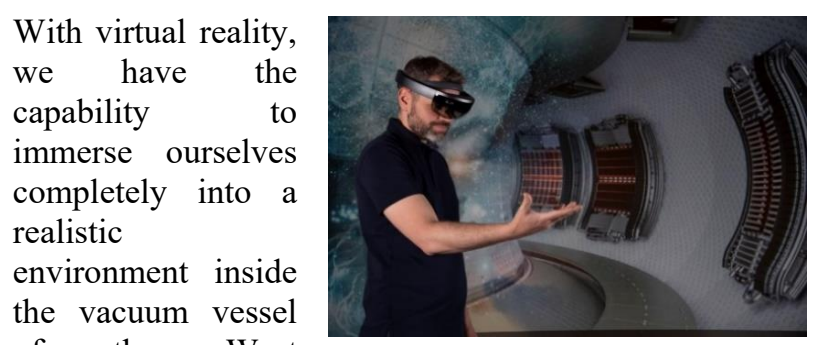
of the West Tokamak. Being Fig.3. WEST virtual environment able to visualise and manipulate virtual elements at true scale holds incredible possibilities into solving problems and is a complete asset into the design process.

With new specific tools such as PIXYZ software, the time process from CAD to VR scene has been drastically reduced using scripting and library data. We can note great improvements in graphic rendering, frame rates and tracking quality on VR devices with the constant evolution of technology.

Although Virtual Reality was already used for static analysis such as design review and clash detection, today expectations can be higher with real time simulations.

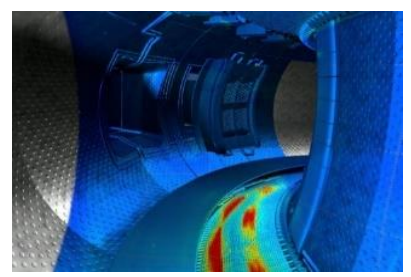

Using light projections in Unity software, we are able to add infrared diagnostic 2D temperature data in a $3 \mathrm{D}$ environment allowing technicians to identify hotspots precisely.

Fig.4. infrared data 3Dviewing

With the "Assembly" module of INTERACT plugin in unity software, environments allows to build real assembly scenarios and check feasibility.

In a near future, artificial intelligence and interconnected IOT will take into account parameters such

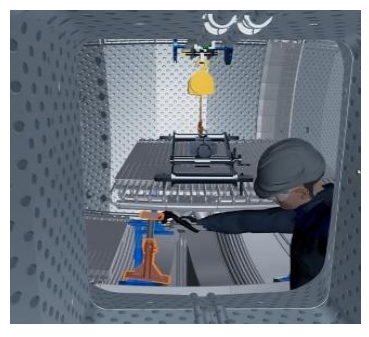

Fig.5. Assembly in VR as emotional state or connected tools data in order to help operators completing the whole assembly sequence.

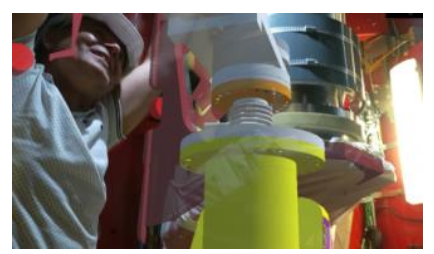

Recently, human access with Augmented Reality is becoming part of the design process.

Microsoft Hololens 2 makes possible to stack

Fig.6. AR on WEST site virtual elements over a real context allowing accessibility studies and design review directly on site. With a $52^{\circ}$ field of view and improved technical characteristics this embedded technology has made a significant progress and becomes interesting for this type of analysis. Further evolution will make AR more accessible with enhanced computations and stronger interaction capabilities.

\section{MIFI : Magnet Infrastructure Facilities for ITER}

Assembling of ITER components is a major challenge considering the large size and weight of components and the high accuracy that has to be reached. As part of the Magnets Infrastructures Facility for ITER (MIFI) agreement between CEA and ITER Organisation, CEA works on the assembly sequence of ITER TF coils OIS (Outer Interface Structure) composed of shear pins and bolts located in a small space which considerably complicates their manipulation and assembling.

A specific tool developed by the CEA, and inspired by tele-manipulators, uses complex kinematics and a counterweight in order to be balanced in any position, allowing the operator to easily manipulate the $35 \mathrm{~kg}$ bolt.

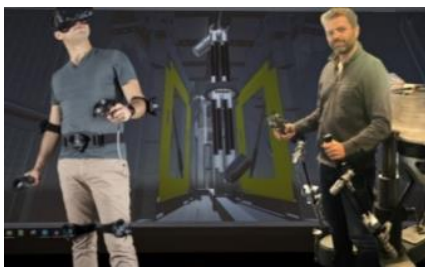

Fig.7. MIFI studies with haptic robotic arm in $V R$
As the assembly sequence and the environment are very constrained, accessibility has been checked thanks to Virtual Reality through immersive scene using contact interaction and force feedback with haptic robotic arms and XDE interactive physics engine developed by CEA-LIST. The operator is able to immerse himself under the coil and manipulate the counterweight trying to fit the pin in his hole with high tolerances. Additional data such as augmented screen streaming camera video in real time are added in order to have a better view of the context.

The handling and positioning tool was also successfully demonstrated on a physical mock-up of the lower part of the coil structure at MIFI [2]

The operator is placed in situation and can manipulate the tool equipped with the counterweight.

AR simulation is added to superimpose the virtual workspace using a Microsoft Hololens 2 device allowing him to apprehend the

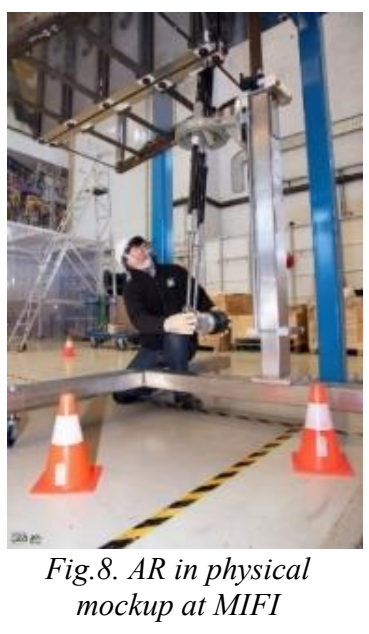


constraint environment.

With iterative studies between design, analysis and simulation using VR/AR we were able to reduce the time to market to design such a complex tool.

\section{$4 \quad$ TBM : Test Blanket Module System}

For ITER Organisation concerning the development of the Test Blanket Modules replacement operations in port cells, CEA develops design recommendation by combination of Virtual Reality Tools and Nuclear Engineering Design activities in Support of Test Blanket Modules Systems development.

To implement ALARA optimisation process since early engineering phase of TBM component, immersive and interactive technologies allows to find ways to optimise component design to minimise occupational exposures of worker, implementing therefore recommendations from International Atomic Energy Agency [9].
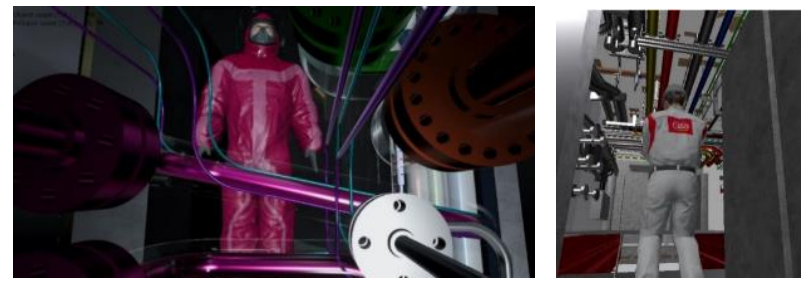

Fig. 9 \& 10. Virtual avatars in TBM Port Cell environment

Several scenario and immersive scenes were developed. During TBM design process, working sessions on VR setup have been organised with ITER, nuclear facility operators, integrators and component designers.

Using virtual avatar equipped with nuclear bubble suit (see fig 9) and advanced operator body tracking, enable to maximise the simulation realism and enhance validation step of TMB tasks by VR.
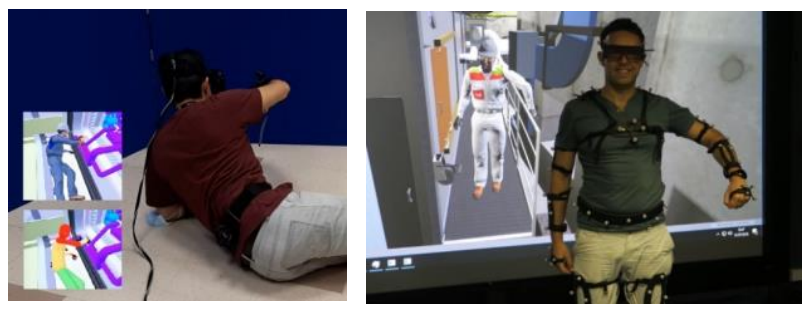

Fig. 11 \& 12. Advanced Real-time Tracking

In view of operator working conditions assessment, we plan to support ergonomics studies thanks to motion capture and force-feedback systems allowing real-time information during design review and take into account Human factor and associated ergonomics requirement.

Cutting \& Welded tools were implemented in the virtual environment with model of interaction and visual feedback such as process effect like production of metal chips.

The use of mixed reality capture in order to record a real user interacting with a virtual world is an asset allowing real time viewing of a VR experience without the use of an avatar.

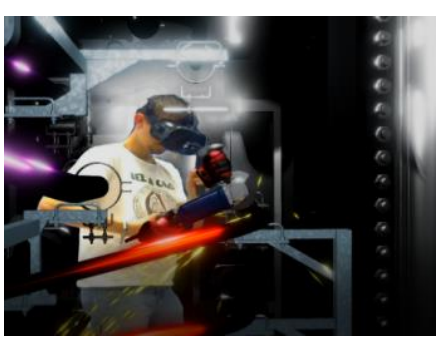

Fig.13. Welding tools integration and Mixed Reality Capture
CEA team also prepared an Augmented Reality simulation in order to study on the workers accessibility. The model is displayed through wearable glasses (Hololens 2) with CEA embedded software features that allows to superimpose real and virtual environment.

This set up has been experimented in situ (IO site, inside the TBM Equatorial Port Cell \#16 before commissioning), in order to compare the virtual model to the real environment enhance immersion conditions. [3]
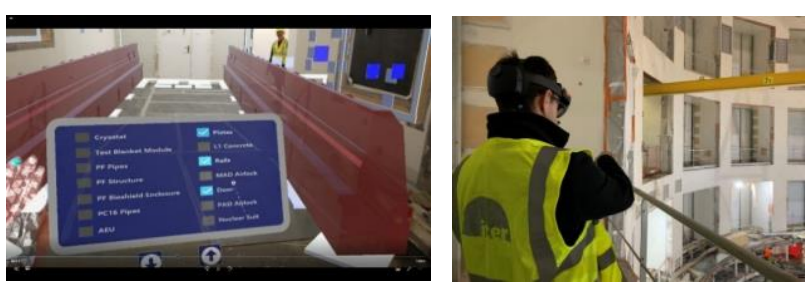

Fig. 14 \& 15. Augmented reality simulation on ITER site

Working sessions allowed confirming the benefits of Augmented Reality in situ, in particular for combined assessment of Design Integration and Health and Safety teams. Human accessibility studies are facilitated by a direct immersion with the real context enhanced with the $3 \mathrm{D}$ hologram of the model.

The accuracy of the virtual/real superimposition is relevant for global checking between models and as built however, enhancement of performance can be expected by proper calibration process and additional features.

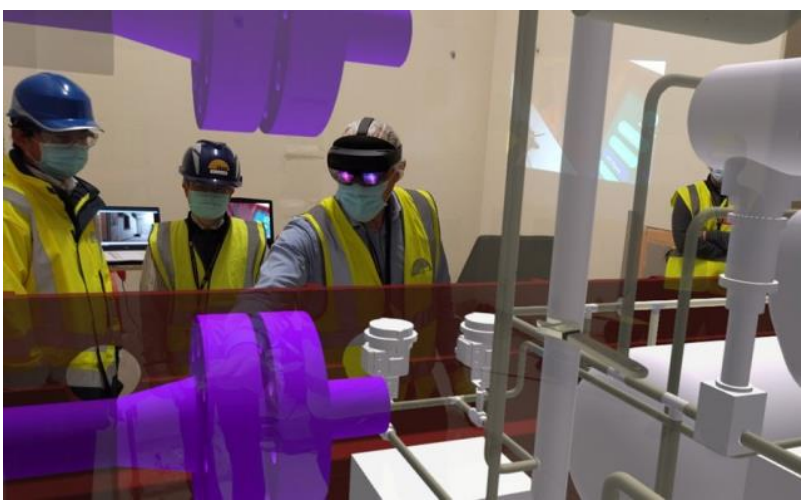

Fig.16. Augmented reality simulation on ITER site 


\section{Conclusion: Next steps and Perspectives}

Following a first thesis on operators, accessibility studies for maintenance scenario [4] future work will focus on qualification of immersive simulation for feasibility studies of human operators.

Several areas of investigation and development are already identified:

- Simulation of the TBM representative replacement task with an operator in bubble suit and umbilical onto a mixed AR \& Physical mock-up.

- Perform design review and plan assembly/reconfiguration sequences in situ in order to gain confidence in the feasibility

- develop adequate user interface for interacting between real and virtual environment

- Include Dose rate elements in AR and VR studies as part of the ALARA process

- Investigate and evaluate Advanced calibration methods considering specific features

- Investigate XR multi users for full intervention scenario (e.g. injured people evacuation)

- Integrate additional model interaction feed back like sounds in the real time simulation

- Develop a TBM Pipes Forest Digital Twin by mechanical behaviour model into VR to simulate displacement based on pipe stress analysis results for replacement sequence assessement and validation

- Virtual commissioning : Augmented/Mixed Reality on physical TBM Pipe Forest Mock-Up

- Coupling physical ergonomic work-bench \& AR

\section{Disclaimer}

The views and opinions expressed herein do not necessarily reflect those of the ITER Organization.

\section{$7 \quad$ References}

[1] L. Meunier et al., Virtual Reality: Lessons Learned from WEST Design and Perspectives for Nuclear Environment, ISFNT-13 (2017).

[2] ITER NEWSLINE - Assembly Zero-gravity in a cramped place 02/2020 https://www.iter.org/newsline/$\underline{13398}$

[3] ITER NEWSLINE - Augmented reality Assessing the future work environment 10/2020 https://www.iter.org/newsline/-/3509

[4] C. Louison et al., Operators' accessibility studies for assembly and maintenance scenarios using virtual reality, Fusion Engineering and Design (2016).

[5] A. Grosman et al., The WEST programme: Minimising technology and operational risks of a full actively cooled tungsten divertor on ITER, Fusion Engineering and Design 88 (2013) 497- 500.

[6] J. Bucalossi et al., Progresses on WEST Platform Construction towards First Plasmas, 26th IAEA Fusion Energy Conference (2016).

[7] B. Peluso, et al., " Magnet Infrastructure Facilities for ITER (MIFI): Description and Activities Overview » IEEE Transactions on Applied Superconductivity (2018) http://ieeexplore.ieee.org/document/8110704/

[8] Giancarli \& al. Overview of recent ITER TBM Program activities. Fusion Engineering and Design 158, 111674. https://doi.org/10.1016/j.fusengdes.2020.111674

[9] Optimisation of Radiation Protection in the Control of Occupational Exposure IAEA2002

[10] https://www.pixyz-software.com/

[11] https://light-and-shadows.com/fr/projet/interact-2/ 